\title{
A randomised controlled trial comparing palate surgery at 6 months versus 12 months of age (the TOPS trial): a statistical analysis plan
}

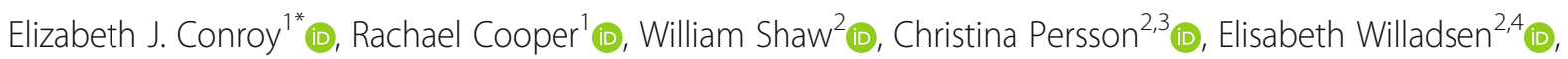
Kevin J. Munro 5,6 (D) Paula R. Williamson ${ }^{1}$ (D), Gunvor Semb², Tanya Walsh², Carrol Gamble ${ }^{1}$ (D) and On behalf of the TOPS trial management group

\begin{abstract}
Background: Cleft palate is among the most common birth abnormalities. The success of primary surgery in the early months of life is crucial for successful feeding, hearing, dental development, and facial growth. Over recent decades, age at palatal surgery in infancy has reduced. The Timing Of Primary Surgery for cleft palate (TOPS) trial aims to determine whether, in infants with cleft palate, it is better to perform primary surgery at age 6 or 12 months (corrected for gestational age).

Methods/design: The TOPS trial is an international, two-arm, parallel group, randomised controlled trial. The primary outcome is insufficient velopharyngeal function at 5 years of age. Secondary outcomes, measured at 12 months, 3 years, and 5 years of age, include measures of speech development, safety of the procedure, hearing level, middle ear function, dentofacial development, and growth. The analysis approaches for primary and secondary outcomes are described here, as are the descriptive statistics which will be reported. The TOPS protocol has been published previously.
\end{abstract}

Discussion: This paper provides details of the planned statistical analyses for the TOPS trial and will reduce the risk of outcome reporting bias and data-driven results.

Trial registration: ClinicalTrials.gov NCT00993551. Registered on 9 October 2009.

Keywords: Randomised controlled trial, Complex intervention, Surgical intervention, Trial design, Trial analysis, Statistical analysis plan, Palatal surgery, Unilateral cleft palate, Sommerlad technique, Velopharyngeal function

\footnotetext{
* Correspondence: ejconroy@liverpool.ac.uk

Related Article: https://doi.org/10.1136/bmjopen-2019-029780

'Liverpool Clinical Trials Centre, University of Liverpool, a member of

Liverpool Health Partners, Institute of Child Health, Alder Hey Children's NHS

Foundation Trust, Liverpool L12 2AP, UK

Full list of author information is available at the end of the article
}

(c) The Author(s). 2021 Open Access This article is licensed under a Creative Commons Attribution 4.0 International License, which permits use, sharing, adaptation, distribution and reproduction in any medium or format, as long as you give appropriate credit to the original author(s) and the source, provide a link to the Creative Commons licence, and indicate if changes were made. The images or other third party material in this article are included in the article's Creative Commons licence, unless indicated otherwise in a credit line to the material. If material is not included in the article's Creative Commons licence and your intended use is not permitted by statutory regulation or exceeds the permitted use, you will need to obtain permission directly from the copyright holder. To view a copy of this licence, visit http://creativecommons.org/licenses/by/4.0/. The Creative Commons Public Domain Dedication waiver (http://creativecommons.org/publicdomain/zero/1.0/) applies to the data made available in this article, unless otherwise stated in a credit line to the data. 


\section{Background}

Clefts of the lip and/or palate are among the most common birth anomalies, occurring with an incidence of 1 in 600 births [1]. The timing of palatal surgery has been a controversial issue since the 1930s [2]. Traditionally, rationale for delaying hard palate surgery was partly based on the belief that postponing the trauma of palatal closure may reduce maxillary growth disturbance. However, there is little evidence that facial skeletal growth in individuals with isolated cleft palate is substantially affected by different surgical protocols, though maxillary arch form, especially transversely, may be affected [3-6].

Over recent decades, the age at which palatal surgery is carried out has reduced. This has led to one-stage palatal closure within 12 months of age at cleft units in Europe and the USA. Protagonists of early closure of the palatal cleft have proposed that since speech is a learnt behaviour, the sooner an intact anatomy is created, the better [7-10]. As yet, however, there is no evidence that early surgery would lead to better speech development.

The Timing of Primary Surgery for cleft palate (TOPS) trial is an international, two-arm, parallel group, randomised controlled trial designed to determine whether, in infants with isolated cleft palate, it is better to perform primary surgery at age 6 or 12 months (corrected for gestational age). This research will investigate the effect of the timing of surgery by assessing and comparing speech development outcomes measured across 12 months, 3 years, and 5 years of age. In addition, secondary outcomes include safety of the procedure, hearing level, middle ear function, dentofacial development, and growth. The protocol paper for the TOPS trial has been published previously [1]; the aim of this paper is to report in detail the statistical analysis plan. This paper has been prepared according to the published guidelines on the content of statistical analysis plans [11].

\section{Methods and design Trial design}

TOPS is an international, multi-centre trial using a parallel arm design aiming to detect whether surgery at 6 months is superior to surgery at 12 months. Infants with a diagnosis of cleft palate are randomised to receive primary surgery for cleft palate using a standardised technique (the Sommerlad technique [12]) at either 6 months or 12 months (corrected for gestational age). Eligible patients are randomised on a 1:1 basis using minimisation routine, incorporating a random element to reduce predictability, to balance the two groups by surgeon $(n=24)$ and size of cleft (soft palate only vs. soft and hard palate). The nature of the intervention prevented this trial from being blind to participants or their carers. However, speech outcomes, at ages 12 months,
3 years, and 5 years, will be rated blind to the randomly allocated group by independent assessment of speech recordings taken at visit. The primary outcome is assessed at age 5 years with secondary outcomes assessed $48 \mathrm{~h}$ and 30 days post-surgery and at age 12 months, 3 years, and 5 years. Full details of the trial design, study population, and study procedures have been published previously [1].

The trial is registered with the ClinicalTrials.gov Identifier: NCT00993551 (registered: 9 October 2009).

\section{Objectives}

The primary objective is to determine whether surgery for cleft palate, using the Sommerlad technique, at age 6 months when compared to surgery at age 12 months improves velopharyngeal function at age 5 years. Secondary research objectives include whether timing of surgery improves speech development, safety of the procedure, hearing level, middle ear function, dentofacial development, and growth.

\section{Outcomes \\ Primary outcome}

The primary outcome is defined as a dichotomous outcome of whether the child has been perceived by Speech and Language Therapists (SLTs), following independent review of speech recordings, to have insufficient velopharyngeal function at age 5 years or not. Velopharyngeal insufficiency is measured by Velopharyngeal Composite Score (VPC) sum, which is a sum of scores, based on three components: hypernasality, non-oral errors, and velopharyngeal insufficiency (VPI) symptoms. Each component is classified and each classification mapped on to a score, see Table 1 . The sum of the three scores, see Eq. 1, gives the VPC sum on the scale 0-6 [13]. Scores $\geq 4$ on this scale will be considered insufficient.

Equation 1: Using the three component scores to calculate VPC sum

$$
\begin{aligned}
\mathrm{VPC} \text { sum }= & \text { Hypernasality score } \\
& + \text { Active non-oral errors score } \\
& + \text { VPI symptoms score }
\end{aligned}
$$

\section{Secondary outcomes}

Secondary outcome measures are defined in the following list. Outcomes 1 to 5 are a measure of speech development, which are classified by SLTs following independent review of speech recordings. Outcomes 6, 7,8 , and 10 are a measure of safety of the procedure, hearing level, middle ear function, and growth respectively and are measured at the relevant follow-up visits. Outcome 9 is a measure of dentofacial development 
Table 1 Calculating the VPC sum

\begin{tabular}{|c|c|c|}
\hline Component & Classification & Score for component \\
\hline \multirow[t]{3}{*}{ Hypernasality } & Within normal limits & 0 \\
\hline & Mild resonance & 1 \\
\hline & Moderate/severe resonance & 2 \\
\hline \multirow[t]{3}{*}{ Non-oral errors } & $0-2$ errors & 0 \\
\hline & $3-5$ errors & 1 \\
\hline & $\geq 6$ errors & 2 \\
\hline \multirow[t]{3}{*}{ Velopharyngeal insufficiency symptoms } & $0-2$ symptoms & 0 \\
\hline & 3-5 symptoms & 1 \\
\hline & $\geq 6$ symptoms & 2 \\
\hline
\end{tabular}

measured independently on a profile photograph and maxillary arch impression taken during the 5-year follow-up visit.

1 Velopharyngeal function at age 5 years:

a Velopharyngeal composite score summary (VPC sum): a long ordinal outcome of individual score that contributes to the primary outcome, see Table 1 . This score is measured on a scale of $0-6$.

b Insufficient velopharyngeal function (VPC rate): a dichotomous outcome of whether the child has "insufficient" VPC rate.

2 Velopharyngeal function at age 3 years:

a Insufficient velopharyngeal function (VPC rate): a dichotomous outcome of whether the child has "insufficient" VPC rate.

b Velopharyngeal insufficiency symptoms: a bounded continuous outcome, the proportion of times that a target consonant uttered has a velopharyngeal insufficiency symptom. Each child will attempt a minimum of 15 and a maximum of 30 predetermined target consonants (in words).

3 Canonical babbling at age 12 months:

a Canonical babbling present: a dichotomous outcome of whether the child is "canonical" or "not canonical".

b Canonical babbling ratio: a bounded continuous outcome, the proportion of times that a syllable produced is "canonical". Determined as the average proportion from the three SLTs undertaking independent review.

c Consonant inventory: a continuous outcome of the number of unique consonants, identified by at least two of three SLTs undertaking independent review, uttered by a child.
4 Articulation at age 3 years: Each child is required to have attempted a minimum of 15 and a maximum of 30 predetermined target consonants (in words) for articulation assessment.

a Percent consonants correct (PCC): a bounded continuous outcome, the proportion of times that a target consonant is uttered correct.

b Percent correct placement (PCP): a bounded continuous outcome, the proportion of times that a target consonant has the correct place of articulation.

c Percent correct manner (PCM): a bounded continuous outcome, the proportion of times that a target consonant has the correct manner of articulation.

d Non-oral consonant errors: a bounded continuous outcome, the proportion of times that a target consonant is realised as a nonoral error.

e Oral consonant errors: a bounded continuous outcome, the proportion of times that a target consonant is realised as an oral error.

5 Articulation at age 5 years: Each child is required to have attempted a minimum of 18 and a maximum of 36 predetermined target consonants (in words).

a PCC: a bounded continuous outcome, the proportion of times that a target consonant is uttered correct.

b PCP: a bounded continuous outcome, the proportion of times that a target consonant has the correct place of articulation.

c PCM: a bounded continuous outcome, the proportion of times that a target consonant has the correct manner of articulation.

d Non-oral consonant errors: a bounded continuous outcome, the proportion of times that a target consonant is realised as a nonoral error. 
e Oral consonant errors: a bounded continuous outcome, the proportion of times that a target consonant is realised as an oral error.

6 Postoperative/long-term complications:

a Dehiscence: a dichotomous outcome of whether the child has a postoperative dehiscence, measured $48 \mathrm{~h}$ and 30 days postoperatively.

b Infection: a dichotomous outcome of whether the child has a postoperative infection, measured $48 \mathrm{~h}$ and 30 days postoperatively.

c Evidence of fistula: a dichotomous outcome of whether the child has a postoperative fistula, assessed as "Yes" or "Probably", measured 30 days postoperatively and at 3 and 5 years of age.

7 Hearing level:

a At 12 months:

i Abnormal Transient Otoacoustic Emission (TEOAE): a dichotomous outcome of whether the child has abnormal TEOAE.

j Abnormal sound field audiometry: a dichotomous outcome of whether the child has abnormal sound field audiometry. Abnormal sound field audiometry is indicated by a measurement of $>30 \mathrm{~dB} \mathrm{HL}$ for at least one of four frequencies tested: $500 \mathrm{~Hz}, 1000 \mathrm{~Hz}, 2000$ $\mathrm{Hz}$, or $4000 \mathrm{~Hz}$.

b At 3 and 5 years:

i Abnormal pure tone audiometry in at least one ear: a dichotomous outcome of whether the child has abnormal pure tone audiometry. If testing by pure tone audiometry is not possible, sound field audiometry can be used in its place. Abnormal audiometry in at least one ear is indicated by a measurement of $>20 \mathrm{~dB}$ HL using the pure tone method, $>25 \mathrm{~dB}$ $\mathrm{HL}$ for sound field, for at least one of four frequencies tested: $500 \mathrm{~Hz}, 1000 \mathrm{~Hz}, 2000$ $\mathrm{Hz}$, or $4000 \mathrm{~Hz}$.

j Abnormal pure tone audiometry in both ears: a dichotomous outcome defined in the same way as secondary outcome $7 \mathrm{bi}$, for patients who have both ears tested and both tested ears indicate abnormal audiometry.

k Severity of better ear: a short ordinal outcome of the severity of the better ear. If testing by pure tone audiometry is not possible, sound field audiometry can be used in its place. Each patient will be classified according to the average score in the better ear if pure tone, or both ears if
Table 2 Classifying severity in better ear

\begin{tabular}{ll}
\hline Average dB HL & Severity \\
\hline$\leq 20 \mathrm{~dB} \mathrm{HL}$ & Normal \\
Between 21 and $40 \mathrm{~dB} \mathrm{HL}$ & Mild \\
Between 41 and $70 \mathrm{~dB} \mathrm{HL}$ & Moderate \\
Between 71 and $95 \mathrm{~dB} \mathrm{HL}$ & Severe \\
$>95 \mathrm{~dB} \mathrm{HL}$ & Profound \\
\hline
\end{tabular}

sound field, across the four frequencies $(500 \mathrm{~Hz}, 1000 \mathrm{~Hz}, 2000 \mathrm{~Hz}$, or $4000 \mathrm{~Hz}$ ) to the categories in Table 2 [14].

8 Middle ear function:

a Flat line tympanogram in at least one ear: a dichotomous outcome of whether the child has flat line tympanogram, assessed at age 12 months, 3 years, and 5 years. Children with either ear measured as "Type B" will be classified as having flat line tympanogram in at least one ear.

b Flat line tympanogram in both ears: a dichotomous outcome of whether the child has flat line tympanogram, assessed at age 12 months, 3 years, and 5 years. Children with both ears measured as "Type B" will be classified as having flat line tympanogram in both ears.

9 Dentofacial development at age 5 years:

a Soft tissue ANB angle: a continuous outcome of the angle between soft tissue nasion (points A and $\mathrm{B}$ ) measured using a profile photograph [15].

b Maxillary arch constriction score: a bounded continuous outcome, measured using the Huddart/Bodenham scoring system, on a maxilliary and mandibular arch impression. A score can range from -24 to 8 and is measured in whole numbers $[16,17]$.

10 Growth at 12 months:

a Nude weight: a continuous outcome, measured in grammes and recorded to the nearest whole number.

b Crown to heel length: a continuous outcome, measured in centimetres and recorded to one decimal place.

c Occipitofrontal circumference: a continuous outcome, measured in centimetres and recorded to one decimal place.

\section{Sample size}

The sample size calculation was based on a test for proportions using a normal approximation: 292 participants per arm will allow a reduction in insufficient velopharyngeal function at 5 years from 40 to $29 \%$ to be detected 
with $80 \%$ power using a chi-squared test (2-sided significance test at 0.05 level). The estimate of $40 \%$ was obtained using data from a pilot trial of 50 5-year-old participants, conducted during the planning period for the grant application [18]. To allow an approximate attrition of 10\%, 648 participants will be recruited. Restating the power for 300 participants per arm will allow the same difference to be detected with $81 \%$ power using a chi-squared test (2-sided significance test at 0.05 level). To consider the potential impact of variability around the value of $40 \%, 300$ participants with valid data per group would provide $80 \%$ power to detect a reduction from 30 to $20 \%$ and $76 \%$ power to detect a reduction from 20 to $12 \%$.

\section{Statistical analysis \\ General analysis principles}

Three analysis populations will be considered: the intention-to-treat (ITT), the per-protocol (PP), and the safety population.

The principle of ITT, as far as practically possible, will be the main strategy of the analysis adopted for the primary outcome and all the secondary outcomes. These analyses will be conducted on all randomised participants, in the group to which they were allocated, and for whom the outcomes of interest have been observed/ measured. No imputations are planned.

A per-protocol analysis, which will mirror the ITT population but exclude participants defined as having a major protocol deviation, will only be considered in the event of major protocol deviations in more than $10 \%$ of the ITT analysis population and apply to a secondary analysis of the primary outcome only. Table S1 provides a list of the protocol deviations.

The safety dataset will classify participants who have surgery before 9 months of gestational corrected age as received 6 months surgery, and surgery at 9 months of gestational corrected age or beyond as received surgery at 12 months.

A $p$ value of 0.05 or less will be used to declare statistical significance for all analyses; $p$ values will be reported to two significant figures. Rather than adjust for multiplicity, relevant results from other studies already reported in the literature will be taken into account when interpreting the study. Percentages will be presented to one decimal place, and continuous summary statistics will be given to a maximum of two decimal places.

All analyses will be performed using standard statistical software (SAS 9.4 or later). The finalised analysis datasets, programs, and outputs will be archived following Good Clinical Practice guidelines and standard operating procedures at the Liverpool Clinical Trials Centre.

\section{Descriptive analyses}

The flow of participants through each stage of the trial, including the number of individuals screened, randomised, receiving treatment as allocated, and included in the primary analysis, will be summarised using a CONSORT flow chart [19] (Fig. 1).

The baseline comparability of the two randomised groups in terms of minimisation factors, demographic characteristics, and clinical genetics will be presented (Table 3).

The surgical comparability of the two randomised groups in terms of baseline surgery characteristics, intraoperative events, early complications during the hospital stay, observations monitored $48 \mathrm{~h}$ post-surgery, and postoperative medication will be presented (Table 4).

Binary and categorical data will be summarised by frequencies and percentages. Continuous data will be presented by means and standard deviations (SDs), or medians and inter-quartile range (IQR) if data are skewed. Tests of statistical significance will not be undertaken for baseline characteristics; rather, the clinical importance of any imbalance will be noted. The amount missing in each case will be summarised.

\section{Lost to follow-up, withdrawals, and missing data}

The timing of withdrawal in relation to surgery and scheduled visits, level of withdrawal, who made the decision, and reason for withdrawal will be summarised both overall and for each randomised group. Frequencies will be presented along with percentages using the number of participants who withdrew as the denominator.

The number lost to follow-up both overall and within each randomised group will be reported, and the reasons where known will be documented. Any deaths and their causes will be reported separately.

Based on experience from the ScandCleft study, the structure of the centralised cleft palate care system and trial-specific systems in place will ensure the occurrence of missing data is likely to be low. Therefore, the potential impact of any missing data is likely to be low. For all assessments and outcomes, participants with insufficient data to make their assessments will be expressed as a frequency and a percentage with the denominator being those who were randomised, treated, and consented.

\section{Adherence}

Reasons for participants not receiving the randomised allocation will be summarised in a table. Adherence with follow-up time points ( 30 days, 12 months, 3 and 5 years) will be summarised at the visit level (at least one scheduled assessment visit completed per time point) and assessment level, which will specify adherence to specific assessments. When applicable, whether or not assessments were made within the expected window (Table 5) 


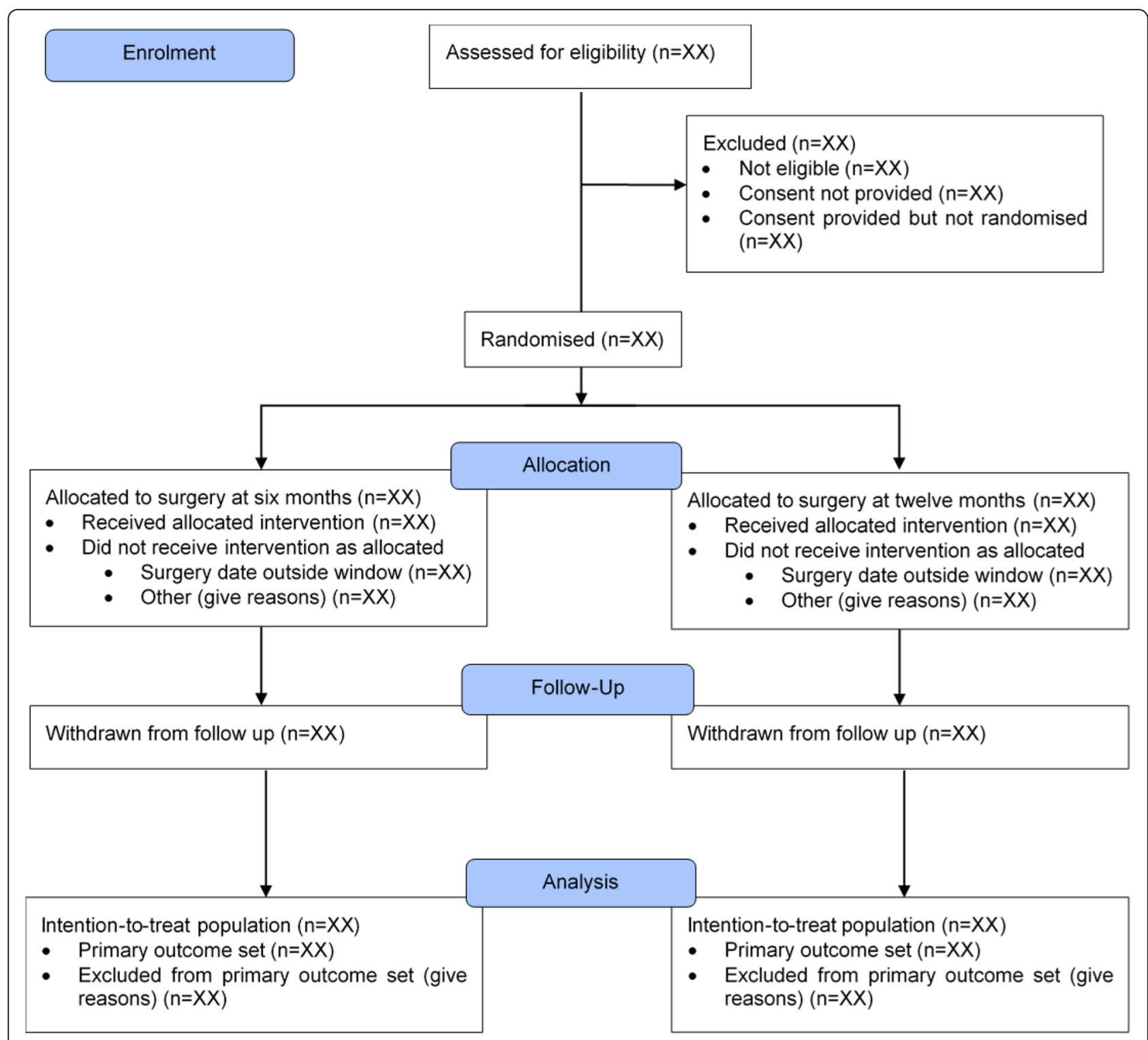

Fig. 1 CONSORT flow diagram for participants in trial up to final assessment

will be presented. Summaries will be presented both overall and for each randomised group.

\section{Analysis of primary outcome}

The number of participants at age 5 years who have the primary outcome of insufficient velopharyngeal function (VPC sum $\geq 4$ ) or not (VPC sum <4) will be summarised overall, for each randomised group and for each region (defined according to the location of the recruiting site: Brazil, Scandinavia, UK) by frequencies and percentages. The numbers of insufficient velopharyngeal function or not between the randomised groups will be compared using a chi-squared test, with the relative risk [20] and 95\% confidence interval (95\% CI) also reported.
In the circumstance that the expected insufficient VPC sum or not for each randomised group contains less than five participants, thereby raising concerns over the appropriateness of a chi-squared test, then Fisher's exact test will be used.

For inclusion in the primary analysis set, a participant must have an eligible 5-year speech recording for SLT assessment. An eligible speech recording requires the child to have attempted at least 18 of the 36 prespecified target words for assessment of non-oral and VPI symptom and 5 of the 9 pre-specified words for hypernasality. Participants who have speech recordings that do not meet the inclusion criteria, have a recording that could not be assessed (e.g. insufficient sound), or 
Table 3 Baseline characteristics and clinical genetics

\begin{tabular}{|c|c|c|c|}
\hline & 6 months surgery & 12 months surgery & Overall \\
\hline Number of patients & $X X$ & $X X$ & $X X$ \\
\hline \multicolumn{4}{|l|}{ Baseline characteristics } \\
\hline \multicolumn{4}{|c|}{ Gender } \\
\hline$N$ & $X X$ & $X X$ & $x X$ \\
\hline Male; $n$ (\%) & $X X(X X . X)$ & $X X(X X . X)$ & $X X(X X . X)$ \\
\hline Female; $n$ (\%) & $X X(X X . X)$ & $X X(X X . X)$ & $X X(X X . X)$ \\
\hline Not known; $n$ & $X X$ & $X X$ & $x X$ \\
\hline \multicolumn{4}{|l|}{ Gestational age (weeks) } \\
\hline$N$ & $x X$ & $X X$ & $x X$ \\
\hline Mean (SD) & $X X . X(X X . X)$ & $X X . X(X X . X)$ & $X X . X(X X . X)$ \\
\hline Median (IQR) & $X X . X(X X . X)$ & $X X . X(X X . X)$ & $X X . X(X X . X)$ \\
\hline (Min, max) & $(X X . X, X X . X)$ & $(X X . X, X X . X)$ & $(X X . X, X X . X)$ \\
\hline Not known; $n$ & $X X$ & $X X$ & $x X$ \\
\hline \multicolumn{4}{|l|}{ Size of cleft } \\
\hline$N$ & $X X$ & $X X$ & $X X$ \\
\hline Soft palate only; $n$ (\%) & $X X(X X . X)$ & $X X(X X . X)$ & $X X(X X . X)$ \\
\hline Soft and hard palate; $n(\%)$ & $X X(X X . X)$ & $X X(X X . X)$ & $X X(X X . X)$ \\
\hline Not known; $n$ & $X X$ & $x X$ & $x X$ \\
\hline \multicolumn{4}{|l|}{ Clinical genetics } \\
\hline \multicolumn{4}{|l|}{ Ethnicity } \\
\hline White; $n(\%)$ & $X X(X X . X)$ & $X X(X X . X)$ & $X X(X X . X)$ \\
\hline Black; n (\%) & $X X(X X . X)$ & $X X(X X . X)$ & $X X(X X . X)$ \\
\hline Asian; $n$ (\%) & $X X(X X . X)$ & $X X(X X . X)$ & $X X(X X . X)$ \\
\hline Chinese; $n$ (\%) & $X X(X X . X)$ & $X X(X X . X)$ & $X X(X X . X)$ \\
\hline Mixed; $n$ (\%) & $X X(X X . X)$ & $X X(X X . X)$ & $X X(X X . X)$ \\
\hline Other; $n$ (\%) & $X X(X X . X)$ & $X X(X X . X)$ & $X X(X X . X)$ \\
\hline Not stated; $n(\%)$ & $X X(X X . X)$ & $X X(X X . X)$ & $X X(X X . X)$ \\
\hline Not known; $n$ & $X X$ & $X X$ & $X X$ \\
\hline \multicolumn{4}{|c|}{ Weight at examination (grammes) } \\
\hline$N$ & $X X$ & $X X$ & $X X$ \\
\hline Mean (SD) & $X X . X(X X . X)$ & $X X . X(X X . X)$ & $X X . X(X X . X)$ \\
\hline Median (IQR) & $X X . X(X X . X)$ & $X X . X(X X . X)$ & $X X . X(X X . X)$ \\
\hline (Min, max) & $(X X . X, X X . X)$ & $(X X . X, X X . X)$ & $(X X . X, X X . X)$ \\
\hline Not known; n & $X X$ & $X X$ & $X X$ \\
\hline \multicolumn{4}{|l|}{ Length at examination $(\mathrm{cm})$} \\
\hline$N$ & $X X$ & $X X$ & $X X$ \\
\hline Mean (SD) & $X X . X(X X . X)$ & $X X . X(X X . X)$ & $X X . X(X X . X)$ \\
\hline Median (IQR) & $X X . X(X X . X)$ & $X X . X(X X . X)$ & $X X . X(X X . X)$ \\
\hline (Min, max) & $(X X . X, X X . X)$ & $(X X . X, X X . X)$ & $(X X . X, X X . X)$ \\
\hline Not known; $n$ & $X X$ & $X X$ & $X X$ \\
\hline \multicolumn{4}{|c|}{ Occipitofrontal circumference $(\mathrm{cm})$} \\
\hline N & $X X$ & $X X$ & $X X$ \\
\hline Mean (SD) & $X X . X(X X . X)$ & $X X . X(X X . X)$ & $X X . X(X X . X)$ \\
\hline
\end{tabular}


Table 3 Baseline characteristics and clinical genetics (Continued)

\begin{tabular}{llll}
\hline & $\mathbf{6}$ months surgery & $\mathbf{1 2}$ months surgery & Overall \\
\hline Median (IQR) & $X X . X(X X . X)$ & $X X . X(X X . X)$ & $(X X . X(X X . X)$ \\
(Min, max) & $(X X . X, X X . X)$ & $(X X . X, X X . X)$ & $X X$ \\
Not known; $n$ & $X X X X . X)$ \\
Interpretation of DENVER-II & & $X X$ & $X X(X X . X)$ \\
Normal; $n$ (\%) & $X X(X X . X)$ & $X X(X X . X)$ & $X X(X X . X)$ \\
Suspect; $n$ (\%) & $X X(X X . X)$ & $X X(X X . X)$ & $X X(X X . X)$ \\
Un-testable; $n$ (\%) & $X X(X X . X)$ & $X X(X X . X)$ & $X X$ \\
Not known; $n$ & $X X$ & $X X$ & $X X(X X . X)$ \\
Diagnosis & & & $X X(X X . X)$ \\
Known syndrome; $n$ (\%) & $X X(X X . X)$ & $X X(X X . X)$ & $X X(X X . X)$ \\
Unknown syndrome; $n$ (\%) & $X X(X X . X)$ & $X X(X X . X)$ & $X X(X X . X)$ \\
Severe developmental delay; $n$ (\%) & $X X(X X . X)$ & $X X(X X . X)$ \\
Uncertain; $n$ (\%) & $X X(X X . X)$ & $X X(X X . X)$ & $X X$ \\
Non-syndromic; $n$ (\%) & $X X(X X . X)$ & $X X(X X . X)$ & \\
Not known; $n$ & $X X X X$ & \\
\hline
\end{tabular}

did not complete a speech recording will be excluded from the analysis.

A sensitivity analysis will be performed to check the robustness of the results to the inclusion/exclusion set for speech recordings such that assessments made on:

- Non-oral and VPI symptom recordings with less than 18 target words attempted are included;

- +Hypernasality recordings with less than 5 target words attempted are included;

- Audio recordings, where video recording not possible, for non-oral and VPI symptom are excluded;

- Any recordings taken outside of the speech recording follow-up window (Table 5) are excluded.

A multilevel logistic regression model (for insufficient VPC sum) adjusting for operating surgeon, size of cleft at baseline (soft palate only vs. soft and hard palate), randomised group, and an intercept will be applied to check the robustness of the results to an unadjusted analysis approach [21-24].

An exploratory analysis will be undertaken where the primary endpoint of insufficient velopharyngeal function, defined as a score of 4-6 on VPC sum, also includes patients who have a secondary surgery due to velopharyngeal insufficiency. This group will be compared to patients who have a score of 0 to 3 on the VPC sum scale and have not received a secondary surgery due to velophyngeal insufficiency. This redefined binary endpoint will be compared using a chisquared test, with the relative risk [20] and 95\% CI also reported.

\section{Analysis of secondary outcomes}

Analysis approaches for each secondary outcome are dependent on the type of outcome; Table 6 provides a summary.

Dichotomous outcomes For outcomes of this type, see Table 6, the number of participants categorised as having, or not having, the outcome of interest will be summarised overall, for each randomised group by frequencies and percentages. The numbers with the outcome between the randomised groups will be compared using a chi-squared test, with the relative risk [20] and 95\% CI also reported.

In the circumstance that the expected number of participants with and without the outcome for each randomised group contains less than five participants, thereby raising concerns over the appropriateness of a chi-squared test, then Fisher's exact test will be used.

No sensitivity analysis will be performed.

Short ordinal outcomes For outcomes of this type, see Table 6, the number of participants who are categorised into each classification of interest will be summarised overall, for each randomised group by frequencies and percentages. The numbers with the outcome between the randomised groups will be compared using a chisquared test for trend.

In the circumstance that the expected number of participants in each classification for each randomised group contains less than five participants, thereby raising concerns over the appropriateness of a chi-squared test for trend, an alternative appropriate analysis approach 
Table 4 Surgery characteristics and observations

\begin{tabular}{|c|c|c|c|}
\hline & 6 months surgery & 12 months surgery & Overall \\
\hline Number of patients & $X X$ & $X X$ & $X X$ \\
\hline \multicolumn{4}{|c|}{ Baseline surgery characteristics } \\
\hline \multicolumn{4}{|c|}{ Grading of cleft at surgery } \\
\hline N & $X X$ & $X X$ & $X X$ \\
\hline Grade1; n (\%) & $X X(X X . X)$ & $X X(X X . X)$ & $X X(X X . X)$ \\
\hline Grade 2; n (\%) & $X X(X X . X)$ & $X X(X X . X)$ & $X X(X X . X)$ \\
\hline Grade 3; n (\%) & $X X(X X . X)$ & $X X(X X . X)$ & $X X(X X . X)$ \\
\hline Grade 4; $n(\%)$ & $X X(X X . X)$ & $X X(X X . X)$ & $X X(X X . X)$ \\
\hline Not known; $n$ & $X X$ & $X X$ & $x X$ \\
\hline \multicolumn{4}{|c|}{ Shape of cleft } \\
\hline N & $X X$ & $X X$ & $x X$ \\
\hline "U" shaped; $n$ (\%) & $X X(X X . X)$ & $X X(X X . X)$ & $X X(X X . X)$ \\
\hline "V" shaped; n (\%) & $X X(X X . X)$ & $X X(X X . X)$ & $X X(X X . X)$ \\
\hline Not known; $n$ & $X X$ & $X X$ & $x X$ \\
\hline \multicolumn{4}{|c|}{ Dimensions of cleft palate (mm) } \\
\hline \multicolumn{4}{|c|}{ Soft tissue width at posterior hard plate } \\
\hline$N$ & $X X$ & $X X$ & $X X$ \\
\hline Mean (SD) & $X X . X(X X . X)$ & $X X . X(X X . X)$ & $X X . X(X X . X)$ \\
\hline Median (IQR) & $X X . X(X X . X)$ & $X X . X(X X . X)$ & $X X . X(X X . X)$ \\
\hline (Min, max) & $(X X . X, X X . X)$ & $(X X . X, X X . X)$ & $(X X . X, X X . X)$ \\
\hline Not known; n & $X X$ & $X X$ & $X X$ \\
\hline \multicolumn{4}{|c|}{ Bony width at posterior hard palate } \\
\hline N & $X X$ & $x X$ & $X X$ \\
\hline Mean (SD) & $X X . X(X X . X)$ & $X X . X(X X . X)$ & $X X . X(X X . X)$ \\
\hline Median (IQR) & $X X . X(X X . X)$ & $X X . X(X X . X)$ & $X X . X(X X . X)$ \\
\hline (Min, max) & $(X X . X, X X . X)$ & $(X X . X, X X . X)$ & $(X X . X, X X . X)$ \\
\hline Not known; $n$ & $X X$ & $X X$ & $X X$ \\
\hline \multicolumn{4}{|c|}{ Width at base of uvula } \\
\hline$N$ & $X X$ & $X X$ & $X X$ \\
\hline Mean (SD) & $X X . X(X X . X)$ & $X X . X(X X . X)$ & $X X . X(X X . X)$ \\
\hline Median (IQR) & $X X . X(X X . X)$ & $X X . X(X X . X)$ & $X X . X(X X . X)$ \\
\hline$($ Min, max) & $(X X . X, X X . X)$ & $(X X . X, X X . X)$ & $(X X . X, X X . X)$ \\
\hline Not known; $n$ & $X X$ & $X X$ & $X X$ \\
\hline \multicolumn{4}{|c|}{ Length of soft palate (distal base of uvula-hard palate) } \\
\hline N & $X X$ & $x X$ & $X X$ \\
\hline Mean (SD) & $X X . X(X X . X)$ & $X X . X(X X . X)$ & $X X . X(X X . X)$ \\
\hline Median (IQR) & $X X . X(X X . X)$ & $X X . X(X X . X)$ & $X X . X(X X . X)$ \\
\hline (Min, max) & $(X X . X, X X . X)$ & $(X X . X, X X . X)$ & $(X X . X, X X . X)$ \\
\hline Not known; $n$ & $X X$ & $X X$ & $X X$ \\
\hline \multicolumn{4}{|l|}{ Intra-operative events } \\
\hline \multicolumn{4}{|c|}{ Blood transfusion during surgery } \\
\hline N & $X X$ & $X X$ & $X X$ \\
\hline Yes; $n(\%)$ & $X X(X X . X)$ & $X X(X X . X)$ & $X X(X X . X)$ \\
\hline
\end{tabular}


Table 4 Surgery characteristics and observations (Continued)

\begin{tabular}{|c|c|c|c|}
\hline & 6 months surgery & 12 months surgery & Overall \\
\hline No; $n(\%)$ & $X X(X X . X)$ & $X X(X X . X)$ & $X X(X X . X)$ \\
\hline Not known; $n$ & $X X$ & $X X$ & $x X$ \\
\hline \multicolumn{4}{|l|}{ Anaesthetic complications } \\
\hline N & $X X$ & $x X$ & $x X$ \\
\hline Yes; $n(\%)$ & $X X(X X . X)$ & $X X(X X . X)$ & $X X(X X . X)$ \\
\hline No; $n$ (\%) & $X X(X X . X)$ & $X X(X X . X)$ & $X X(X X . X)$ \\
\hline Not known; $n$ & $X X$ & $x X$ & $x X$ \\
\hline \multicolumn{4}{|l|}{ Bleeding } \\
\hline N & $X X$ & $X X$ & $x X$ \\
\hline Yes; $n(\%)$ & $X X(X X . X)$ & $X X(X X . X)$ & $X X(X X . X)$ \\
\hline No; $n(\%)$ & $X X(X X . X)$ & $X X(X X . X)$ & $X X(X X . X)$ \\
\hline Not known; $n$ & $X X$ & $X X$ & $X X$ \\
\hline \multicolumn{4}{|l|}{ Early complications during the hospital stay } \\
\hline \multicolumn{4}{|l|}{ Postoperative airway problems } \\
\hline$N$ & $X X$ & $X X$ & $x X$ \\
\hline Yes; n (\%) & $X X(X X . X)$ & $X X(X X . X)$ & $X X(X X . X)$ \\
\hline No; $n(\%)$ & $X X(X X . X)$ & $X X(X X . X)$ & $X X(X X . X)$ \\
\hline Not known; $n$ & $X X$ & $X X$ & $X X$ \\
\hline \multicolumn{4}{|l|}{ Postoperative blood loss } \\
\hline$N$ & $X X$ & $X X$ & $x X$ \\
\hline Yes; n (\%) & $X X(X X . X)$ & $X X(X X . X)$ & $X X(X X . X)$ \\
\hline No; $n(\%)$ & $X X(X X . X)$ & $X X(X X . X)$ & $X X(X X . X)$ \\
\hline Not known; $n$ & $X X$ & $X X$ & $X X$ \\
\hline \multicolumn{4}{|l|}{ Anti-coagulants given } \\
\hline$N$ & $X X$ & $X X$ & $X X$ \\
\hline Yes; $n(\%)$ & $X X(X X . X)$ & $X X(X X . X)$ & $X X(X X . X)$ \\
\hline No; $n(\%)$ & $X X(X X . X)$ & $X X(X X . X)$ & $X X(X X . X)$ \\
\hline Not known; $n$ & $X X$ & $x X$ & $x X$ \\
\hline \multicolumn{4}{|l|}{ Dehiscence } \\
\hline$N$ & $X X$ & $x X$ & $x X$ \\
\hline Yes; $n(\%)$ & $X X(X X . X)$ & $X X(X X . X)$ & $X X(X X . X)$ \\
\hline No; $n(\%)$ & $X X(X X . X)$ & $X X(X X . X)$ & $X X(X X . X)$ \\
\hline Not known; n & $X X$ & $X X$ & $X X$ \\
\hline \multicolumn{4}{|l|}{ Readmission to operating room } \\
\hline N & $X X$ & $X X$ & $X X$ \\
\hline Yes; $n(\%)$ & $X X(X X . X)$ & $X X(X X . X)$ & $X X(X X . X)$ \\
\hline No; $n(\%)$ & $X X(X X . X)$ & $X X(X X . X)$ & $X X(X X . X)$ \\
\hline Not known; $n$ & $X X$ & $X X$ & $X X$ \\
\hline \multicolumn{4}{|c|}{ Observations monitored in first $48 \mathrm{~h}$ post-surgery } \\
\hline \multicolumn{4}{|l|}{ Oxygen saturation levels } \\
\hline$N$ & $X X$ & $X X$ & $X X$ \\
\hline Clinically significant abnormality; n (\%) & $X X(X X . X)$ & $X X(X X . X)$ & $X X(X X . X)$ \\
\hline No clinically significant abnormality; $n$ (\%) & $X X(X X . X)$ & $X X(X X . X)$ & $X X(X X . X)$ \\
\hline No observation; $n$ (\%) & $X X(X X . X)$ & $X X(X X . X)$ & $X X(X X . X)$ \\
\hline
\end{tabular}


Table 4 Surgery characteristics and observations (Continued)

\begin{tabular}{|c|c|c|c|}
\hline & 6 months surgery & 12 months surgery & Overall \\
\hline Not known; $n$ & $X X$ & $x X$ & $X X$ \\
\hline \multicolumn{4}{|l|}{ Carbon dioxide and oxygen } \\
\hline N & $X X$ & $X X$ & $X X$ \\
\hline Clinically significant abnormality; $n$ (\%) & $X X(X X . X)$ & $X X(X X . X)$ & $X X(X X . X)$ \\
\hline No clinically significant abnormality; $n$ (\%) & $X X(X X . X)$ & $X X(X X . X)$ & $X X(X X . X)$ \\
\hline No observation; $n(\%)$ & $X X(X X . X)$ & $X X(X X . X)$ & $X X(X X . X)$ \\
\hline Not known; $n$ & $x X$ & $x X$ & $X X$ \\
\hline \multicolumn{4}{|l|}{ Arterial blood gases } \\
\hline N & $x X$ & $X X$ & $X X$ \\
\hline Clinically significant abnormality; $n$ (\%) & $X X(X X . X)$ & $X X(X X . X)$ & $X X(X X . X)$ \\
\hline No clinically significant abnormality; $n$ (\%) & $X X(X X . X)$ & $X X(X X . X)$ & $X X(X X . X)$ \\
\hline No observation; $n$ (\%) & $X X(X X . X)$ & $X X(X X . X)$ & $X X(X X . X)$ \\
\hline Not known; $n$ & $X X$ & $X X$ & $X X$ \\
\hline \multicolumn{4}{|l|}{ Heart rate } \\
\hline N & $x X$ & $x X$ & $X X$ \\
\hline Clinically significant abnormality and treatment required; $n$ (\%) & $X X(X X . X)$ & $X X(X X . X)$ & $X X(X X . X)$ \\
\hline Clinically significant abnormality and no treatment required; $n$ (\%) & $X X(X X . X)$ & $X X(X X . X)$ & $X X(X X . X)$ \\
\hline No clinically significant abnormality; $n$ (\%) & $X X(X X . X)$ & $X X(X X . X)$ & $X X(X X . X)$ \\
\hline No observation; $n$ (\%) & $X X(X X . X)$ & $X X(X X . X)$ & $X X(X X . X)$ \\
\hline Not known; $n$ & $x X$ & $x X$ & $X X$ \\
\hline \multicolumn{4}{|l|}{ Blood pressure } \\
\hline N & $x X$ & $X X$ & $X X$ \\
\hline Clinically significant abnormality and treatment required; $n$ (\%) & $X X(X X . X)$ & $X X(X X . X)$ & $X X(X X . X)$ \\
\hline Clinically significant abnormality and no treatment required; $n$ (\%) & $X X(X X . X)$ & $X X(X X . X)$ & $X X(X X . X)$ \\
\hline No clinically significant abnormality; $n$ (\%) & $X X(X X . X)$ & $X X(X X . X)$ & $X X(X X . X)$ \\
\hline No observation; $n$ (\%) & $X X(X X . X)$ & $X X(X X . X)$ & $X X(X X . X)$ \\
\hline Not known; $n$ & $X X$ & $X X$ & $X X$ \\
\hline \multicolumn{4}{|l|}{ Respiration } \\
\hline N & $x X$ & $X X$ & $X X$ \\
\hline Clinically significant abnormality; $n$ (\%) & $X X(X X . X)$ & $X X(X X . X)$ & $X X(X X . X)$ \\
\hline No clinically significant abnormality; $n$ (\%) & $X X(X X . X)$ & $X X(X X . X)$ & $X X(X X . X)$ \\
\hline No observation; $n$ (\%) & $X X(X X . X)$ & $X X(X X . X)$ & $X X(X X . X)$ \\
\hline Not known; $n$ & $X X$ & $X X$ & $X X$ \\
\hline \multicolumn{4}{|l|}{ Body temperature } \\
\hline N & $x X$ & $x X$ & $X X$ \\
\hline Clinically significant abnormality and treatment required; $n$ (\%) & $X X(X X . X)$ & $X X(X X . X)$ & $X X(X X . X)$ \\
\hline Clinically significant abnormality and no treatment required; $n$ (\%) & $X X(X X . X)$ & $X X(X X . X)$ & $X X(X X . X)$ \\
\hline No clinically significant abnormality; $n$ (\%) & $X X(X X . X)$ & $X X(X X . X)$ & $X X(X X . X)$ \\
\hline No observation; $n$ (\%) & $X X(X X . X)$ & $X X(X X . X)$ & $X X(X X . X)$ \\
\hline Not known; $n$ & $X X$ & $X X$ & $X X$ \\
\hline \multicolumn{4}{|l|}{ Postoperative medication } \\
\hline \multicolumn{4}{|l|}{ Pain relief medication } \\
\hline N & $x X$ & $X X$ & $X X$ \\
\hline Yes; $n(\%)$ & $X X(X X . X)$ & $X X(X X . X)$ & $X X(X X . X)$ \\
\hline
\end{tabular}


Table 4 Surgery characteristics and observations (Continued)

\begin{tabular}{llll}
\hline & 6 months surgery & $\mathbf{1 2}$ months surgery & Overall \\
\hline No; $n$ (\%) & $X X(X X . X)$ & $X X(X X . X)$ & $X X(X X . X)$ \\
Not known; $n$ & $X X$ & $X X$ & \\
Anti-inflammatory medication & & $X X$ \\
N & $X X$ & $X X$ & $X X(X X . X)$ \\
Yes; $n$ (\%) & $X X(X X . X)$ & $X X(X X . X)$ & $X X(X X . X)$ \\
No; $n$ (\%) & $X X(X X . X)$ & $X X(X X . X)$ & $X X$ \\
Not known; & $X X$ & $X X$ & $X X$ \\
Antibiotic medication (other than prophylactic antibiotics) & & & \\
N & $X X$ & $X X$ & $X X(X X . X)$ \\
Yes; $n$ (\%) & $X X(X X . X)$ & $X X(X X . X)$ & $X X(X X . X)$ \\
No; $n$ (\%) & $X X(X X . X)$ & $X X(X X . X)$ & $X X$ \\
Not known; $n$ & $X X$ & $X X$ & $X$ \\
\hline
\end{tabular}

will be used, e.g. combining like groups or applying a proportional odds model.

No sensitivity analysis will be performed.

Long ordinal, bounded continuous, and continuous outcomes Outcomes of this type, see Table 6, will be summarised overall and for each randomised group by means and SDs, or medians and IQRs if data are skewed. Minimum and maximum values will also be presented.

Means will be compared between the two randomised groups using a $t$ test or by using a non-parametric equivalent. Testing for normality of data distributions will be based using a QQ plot by randomised group. Ninety-five percent confidence intervals will be presented around the effect measure.

No sensitivity analysis will be performed.

\section{Inter- and intra-rater reliability}

Each of the outcomes assessed by independent assessors, i.e. not at routine visit (outcomes 1-5 and 9), will each be reviewed by a minimum of one assessor and a maximum of three. Intra and inter assessments will be undertaken for a proportion of all outcomes to ensure reliability of the outcome measures. The number of assessors and proportion of inter- and intra-rater assessments is determined a priori and decided on a peroutcome basis, led by members of the Trial Management Group (see http://www.tops-trial.org.uk/) who are specialists in the specific outcome field.

Agreement analysis will be exploratory and report agreement as frequencies and percentages or using Bland-Altman agreement analysis as appropriate for the outcome type [25].

\section{Additional analyses}

To support interpretation of the main trial outcomes, descriptive statistics will be summarised to report the results of the following: (i) the DENVER-II test at 3 years, (ii) additional speech therapy received outside of routine trial visits, (iii) reasons and the nature of any secondary surgeries received during the trial, and (iv) nasometry at 5 years. Binary and categorical data will be summarised

Table 5 Follow-up visits: estimated visit dates and corresponding window limits

\begin{tabular}{|c|c|c|c|}
\hline \multirow{2}{*}{$\begin{array}{l}\text { Time } \\
\text { point }\end{array}$} & \multirow{2}{*}{$\begin{array}{l}\text { Estimated } \\
\text { visit date, } \\
\text { calculated } \\
\text { from date } \\
\text { of birth }\end{array}$} & \multicolumn{2}{|c|}{ Window limits of estimated visit date } \\
\hline & & Earliest & Latest \\
\hline \multirow[t]{2}{*}{12 months } & 52 weeks $^{1}$ & $\begin{array}{l}6 \text { months arm: } \\
-4 \text { weeks }\end{array}$ & +48 weeks (equivalent to 23 months of age) ${ }^{2}$ \\
\hline & & $\begin{array}{l}12 \text { months arm: } \\
-2 \text { weeks }\end{array}$ & \\
\hline 3 years & 156 weeks & -4 weeks & +48 weeks (equivalent to 3 years 11 months of age) \\
\hline 5 years & 260 weeks & -4 weeks & +48 weeks (equivalent to 5 years 11 months of age) \\
\hline
\end{tabular}

${ }^{1}$ Corrected for gestational age. Full term is defined as day 1 of the 40th week of pregnancy

${ }^{2}$ Speech assessments vary in their latest acceptable date:

- Six months surgery patients should have their speech assessed by +26 weeks (equivalent to 18 months of age)

- Twelve months surgery should have their speech assessed before surgery 
Table 6 Endpoint type for secondary outcomes

\begin{tabular}{|c|c|}
\hline Outcome type & Outcome \\
\hline \multirow[t]{18}{*}{ Dichotomous } & 1b: Insufficient velopharyngeal function (VPC rate) at 5 years \\
\hline & 2a: Insufficient velopharyngeal function (VPC rate) at 3 years \\
\hline & 3a: Canonical babbling present at 12 months \\
\hline & 6a: Postoperative dehiscence \\
\hline & 6b: Postoperative infection \\
\hline & 6c: Evidence of fistula \\
\hline & 7ai: Abnormal Transient Otoacoustic Emission (TEOAE) at 12 months \\
\hline & 7aii: Abnormal sound field audiometry at 12 months \\
\hline & 7bi_1: Abnormal pure tone audiometry in at least one ear at 3 years \\
\hline & 7bi_2: Abnormal pure tone audiometry in at least one ear at 5 years \\
\hline & 7bii_1: Abnormal pure tone audiometry in both ears at 3 years \\
\hline & 7bii_2: Abnormal pure tone audiometry in both ears at 5 years \\
\hline & 8a_1: Flat line tympanogram in at least one ear at 12 months \\
\hline & 8a_2: Flat line tympanogram in at least one ear at 3 years \\
\hline & 8a_3: Flat line tympanogram in at least one ear at 5 years \\
\hline & 8b_1: Flat line tympanogram in both ears at 12 months \\
\hline & 8b_2: Flat line tympanogram in both ears at 3 years \\
\hline & 8b_3: Flat line tympanogram in both ears at 5 years \\
\hline \multirow[t]{2}{*}{ Short ordinal } & 7biii_1: Severity of better ear at 3 years \\
\hline & 7biii_2: Severity of better ear at 5 years \\
\hline Long ordinal & 1a: Velopharyngeal composite score summary (VPC sum) at 5 years \\
\hline \multirow[t]{13}{*}{ Bounded continuous } & 2b: Velopharyngeal insufficiency symptoms at 3 years \\
\hline & 3b: Canonical babbling ratio at 12 months \\
\hline & 4a: Percent consonants correct (PCC) at 3 years \\
\hline & 4b: Percent correct placement (PCP) at 3 years \\
\hline & 4c: Percent correct manner (PCM) at 3 years \\
\hline & 4d: Non-oral consonant errors at 3 years \\
\hline & 4e: Oral consonant errors at 3 years \\
\hline & 5a: Percent consonants correct (PCC) at 5 years \\
\hline & 5b: Percent correct placement (PCP) at 5 years \\
\hline & $5 \mathrm{c}$ : Percent correct manner (PCM) at 5 years \\
\hline & $5 \mathrm{~d}$ : Non-oral consonant errors at 5 years \\
\hline & 5e: Oral consonant errors at 5 years \\
\hline & 9b: Maxillary arch constriction score at 5 years \\
\hline \multirow[t]{5}{*}{ Continuous } & 3c: Consonant inventory at 12 months \\
\hline & 9a: Soft tissue ANB angle at 5 years \\
\hline & 10a: Nude weight at 12 months \\
\hline & 10b: Crown to heel length at 12 months \\
\hline & 10c: Occipitofrontal circumference at 12 months \\
\hline
\end{tabular}

by frequencies and percentages. Continuous data will be presented by means and SDs, or medians and IQR if data are skewed.

Tests of statistical significance will not be undertaken for (i), (ii), and (iii); rather, the clinical importance of any imbalance will be noted. The amount missing in each case will be summarised. (iv) Nasometry, at age 5 years, will be compared between the two randomised groups using a $t$ test or by using a non-parametric equivalent. Testing for normality of data distributions 
will be based using a QQ plot by randomised group. Ninety-five percent confidence intervals will be presented around the effect measure.

\section{Safety evaluations}

Serious adverse events and unanticipated problems will be presented using descriptive statistics. Line listings of events will also be presented to provide further detail. Patients will be reported according to the safety dataset, with the number of events and patients in each safety group summarised. Tests of statistical significance will not be undertaken; rather, the clinical importance of any imbalance will be noted.

\section{Discussion}

The TOPS trial will provide evidence to support whether surgery for cleft palate at age 6 months when compared to surgery at age 12 months improves velopharyngeal function at age 5 years. In addition, evidence regarding a wide range of pre-defined clinical secondary outcomes will be explored. This paper provides details of the planned statistical analyses of the trial. Publishing these plans prior to trial results will improve the scientific validity of the TOPS trial and reduce the risk of outcome reporting bias and data-driven results [26].

\section{Trial status}

The trial completed recruitment on 21 July 2015. In total, 558 patients from 22 centres were recruited and the last patient is due to attend their last visit on 30 July 2020. The analysis of outcomes will be conducted thereafter.

\section{Supplementary Information}

The online version contains supplementary material available at https://doi. org/10.1186/s13063-020-04886-y.

Additional file 1: Table S1. Definition of protocol deviations.

\section{Abbreviations \\ 95\% Cl: 95\% confidence interval; IQR: Inter-quartile range; ITT: Intention-to- treat; PCC: Percent consonants correct; PCM: Percent correct manner; PCP: Percent consonants placement; PP: Per-protocol; SD: Standard deviation; SLT: Speech and Language Therapist; TOPS: Timing Of Primary Surgery for cleft palate; VPC: Velopharyngeal Composite Score; VPC rate: Insufficient velopharyngeal function; VPC sum: Velopharyngeal Composite Score summary}

\footnotetext{
Acknowledgements

The authors acknowledge our partners in Cleft Care centres in UK, Scandinavia, and Brazil for participating in data collection, the Speech and Language Therapists for their contributions in calibration and rating of speech assessments during various stages of the TOPS trial, and the TOPS trial management group members (see http://www.tops-trial.org. uk/) and the oversight committee members for reviewing the statistical analysis plan prior to sign off.
}

\section{Authors' contributions}

EJC, RC, WS, and CG drafted and finalised the statistical analysis plan. WS, CP, EW, and KM provided clinical advice with regard to the interpretation and derivation of the outcomes. EJC, CG, and PRW wrote the statistical analysis sections in the trial protocol. WS is the chief investigator for this trial. WS, CP EW, PRW, GS, and CG contributed to the study design and developed the first version of the protocol. All authors read and approved the manuscript for publication.

\section{Funding}

This publication was made possible by grant numbers R21DE15128, U01DE018664, and U01DE018837 from the National Institute of Dental and Craniofacial Research (NIDCR). Its contents are solely the responsibility of the authors and do not necessarily represent the official views of the NIDCR. The funders had no role in the study design, data collection, analysis proposal, or writing of this manuscript.

\section{Availability of data and materials}

Not applicable.

\section{Ethics approval and consent to participate}

The protocol was approved from the Multicentre Research Ethics Committee in the UK (MREC number: 09/H1306/119). Ethical approval has been sought in each participating country according to country-specific procedures and from relevant ethics committees for each participating centre. The trial is registered with ClinicalTrials.gov (Identifier: NCT00993551).

\section{Consent for publication}

Not applicable.

\section{Competing interests}

The authors have no competing interest to disclose.

\section{Author details}

${ }^{1}$ Liverpool Clinical Trials Centre, University of Liverpool, a member of Liverpool Health Partners, Institute of Child Health, Alder Hey Children's NHS Foundation Trust, Liverpool L12 2AP, UK. ${ }^{2}$ School of Medical Sciences, Division of Dentistry, The University of Manchester, Manchester, UK. ${ }^{3}$ Institute of Neuroscience and Physiology, Speech and Language Pathology Unit, Sahlgrenska Academy, University of Gothenburg, Gothenburg, Sweden. ${ }^{4}$ Department of Nordic Studies and Linguistics, University of Copenhagen, Copenhagen, Denmark. ${ }^{5}$ Manchester Centre for Audiology and Deafness, School of Health Sciences, The University of Manchester, Manchester, UK. ${ }^{6}$ Manchester University Hospitals NHS Foundation Trust, Manchester Academic Health Science Centre, Manchester, UK.

Received: 30 March 2020 Accepted: 11 November 2020

Published online: 04 January 2021

\section{References}

1. Shaw W, Semb G, Lohmander A, et al. Timing Of Primary Surgery for cleft palate (TOPS): protocol for a randomised trial of palate surgery at 6 months versus 12 months of age. BMJ Open. 2019;9:e029780. https://doi.org/10. 1136/bmjopen-2019-029780.

2. Peterson-Falzone S. Optimal age for palatoplasty to faclitate normal speech development: what is the evidence? In: Berkowitz S, editor. Cleft lip and palate. Berlin: Springer; 2006. p. 691-700.

3. Hellquist R, Ponten B, Skoog T. The influence of cleft length and palatoplasty on the dental arch and the deciduous occlusion in cases of clefts of the secondary palate. Scand J Plast Reconstr Surg. 1978;12(1):45-54.

4. Friede $H$, Persson EC, Lilja J, et al. Maxillary dental arch and occlusion in patients with repaired clefts of the secondary palate. Influence of push back palatal surgery. Scand J Plast Reconstr Surg Hand Surg / Nordisk plastikkirurgisk forening [and] Nordisk klubb for handkirurgi. 1993;27(4):297-305.

5. Nystrom M, Ranta R. Effect of timing and method of closure of isolated cleft palate on development of dental arches from 3 to 6 years of age. Eur J Orthod. 1994;16(5):377-83.

6. Friede $H$, Enocson L, Moller M, et al. Maxillary dental arch and occlusion in repaired clefts of the secondary palate: influence of surgical closure with minimal denudation of bone. Scand J Plast Reconstr Surg Hand Surg / 
Nordisk plastikkirurgisk forening [and] Nordisk klubb for handkirurgi. 2000; 34(3):213-8.

7. Dorf DS, Curtin JW. Early cleft palate repair and speech outcome. Plast Reconstr Surg. 1982;70(1):74-81.

8. Chapman KL, Hardin-Jones MA, Goldstein JA, et al. Timing of palatal surgery and speech outcome. Cleft Palate Craniofac J. 2008;45(3):297-308. https:// doi.org/10.1597/06-244 [published Online First: Epub Date].

9. Chapman KL, Willadsen E. The development of speech in children with cleft palate. In: Cleft palate speech: assessment and intervention; 2011. p. 23-40. https://doi.org/10.1002/9781118785065. [published Online First: Epub Date].

10. Jones DL. Timing of palatoplasty and speech. In: Comprehensive Cleft Care: Taylor \& Francis Group, LLC. 2016:521-6. https://bmjopen.bmj.com/content/ 9/7/e029780\#xref-ref-7-1.

11. Gamble C, Krishan A, Stocken D, et al. Guidelines for the content of statistical analysis plans in clinical trials. JAMA. 2017;318(23):2337-43. https:// doi.org/10.1001/jama.2017.18556.

12. Sommerlad BC. A technique for cleft palate repair. Plast Reconstr Surg. 2003: 112(6):1542-8. https://doi.org/10.1097/01.PRS.0000085599.84458.D2 [published Online First: Epub Date].

13. Lohmander A, Persson C, Willadsen E, et al. Scandcleft randomised trials of primary surgery for unilateral cleft lip and palate: 4 . Speech outcomes in 5year-olds - velopharyngeal competency and hypernasality. J Plast Surg Hand Surg. 2017;51(1):27-37. https:/doi.org/10.1080/2000656X.2016.1254645 [published Online First: Epub Date].

14. British Society of Audiology. Recommended Procedure: Pure-tone air conduction and bone-conduction threshold audiometry with and without masking. 2011

15. Bearn D, Sandy JR, Shaw WC. Photogrammetric assessment of the soft tissue profile in unilateral cleft lip and palate. Cleft Palate Craniofac J. 2002; 39:597-603.

16. Martin $C B, M a X$, Mclntyre GT, et al. The validity and reliability of an automated method of scoring dental arch relationships in unilateral cleft lip and palate using the modified Huddart-Bodenham scoring system. Eur J Orthod. 2016;38:353-8.

17. Gray D, Mossey PA. Evaluation of a modified Huddart/Bodenham system for assessment of maxillary constriction. Eur J Orthod. 2005;27:507-11.

18. Lohmander A, Willadsen E, Persson C, et al. Methodology for speech assessment in the Scandcleft project-an international randomized clinical trials on palatal surgery: experiences from a pilot study. Cleft Palate Craniofac J. 2009;46:347-62

19. Schulz KF, Altman DG, Moher D, for the CONSORT Group. CONSORT 2010 Statement updated guildelines for reporting parallel group randomized trials. BMJ. 2010;340:c332.

20. Morris JA, Gardner MJ. Calculating confidence intervals for relative risks (odds ratios) and standardised ratios and rates. Stat Med. 1988;296:1313-6.

21. Kahan BC, Morris TP. Assessing potential sources of clustering in individually randomised trials. BMC Med Res Methodol. 2013;13:58. https://doi.org/10. 1186/1471-2288-13-58

22. Kahan BC, Morris TP. Improper analysis of trials randomised using stratified blocks or minimisation. Stat Med. 2012;31:328-40. https://doi. org/10.1002/sim.4431.

23. Hernandez AV, Steyerberg EW, Habbema JD. Covariate adjustment in randomized controlled trials with dichotomous outcomes increases statistical power and reduces sample size requirements. J Clin Epidemiol. 2004;57:454-60. https://doi.org/10.1016/j.jclinepi.2003.09.014.

24. Pocock SJ, Assmann SE, Enos LE, Kasten LE. Subgroup analysis, covariate adjustment and baseline comparisons in clinical trial reporting: current practice and problems. Stat Med. 2002;21:2917-30. https://doi.org/10. 1002/sim.1296.

25. Bland JM, Altman DG. Measuring agreement in method comparison studies. Stat Methods Med Res. 1999:8:135-60. https://doi.org/10.1191/ 096228099673819272.

26. Finfer S, Bellomo R. Why publish statistical analysis plans. Crit Care Resusc. 2009;11(1):5-6.

\section{Publisher's Note}

Springer Nature remains neutral with regard to jurisdictional claims in published maps and institutional affiliations.

\section{Ready to submit your research? Choose BMC and benefit from:}

- fast, convenient online submission

- thorough peer review by experienced researchers in your field

- rapid publication on acceptance

- support for research data, including large and complex data types

- gold Open Access which fosters wider collaboration and increased citations

- maximum visibility for your research: over $100 \mathrm{M}$ website views per year

At BMC, research is always in progress.

Learn more biomedcentral.com/submissions 